\title{
Optomechanical detection of vibration modes of a single bacterium
}

\author{
Eduardo Gil-Santos*1, Jose J. Ruz, Oscar Malvar ${ }^{1}$, Ivan Favero ${ }^{2}$, Aristide Lemaître ${ }^{3}$, Priscila. M. Kosaka ${ }^{1}$, \\ Sergio García-López ${ }^{1}$, Montserrat Calleja ${ }^{1}$, Javier Tamayo*1 \\ ${ }^{1}$ Bionanomechanics Lab, Instituto de Micro y Nanotecnología, IMN-CNM (CSIC), Isaac Newton 8 (PTM), E- \\ 28760 Tres Cantos, Madrid, Spain. \\ ${ }^{2}$ Matériaux et Phénomènes Quantiques, Université de Paris, CNRS, 10 rue Alice Domon et Léonie Duquet, \\ 75013 Paris, France. \\ ${ }^{3}$ Centre de Nanosciences et Nanotechnologies, CNRS, Université Paris-Saclay, 10 boulevard Thomas Gobert, \\ 91120 Palaiseau, France. \\ Correspondence to: jtamayo@imm.cnm.csic.es, eduardo.gil@csic.es
}

\section{Abstract:}

Low-frequency vibration modes of biological particles such as proteins, viruses and bacteria involve coherent collective vibrations at frequencies in the terahertz and gigahertz domains. These vibration modes carry information on their structure and mechanical properties, which are good indicators of their biological state. In this work, we harness a particular regime in the physics of coupled mechanical resonators to directly measure the mechanical resonances of single bacterium. We deposit the bacterium on the surface of an ultra-high frequency optomechanical disk resonator in ambient conditions. The vibration modes of the disk and bacterium hybridize when their associated frequencies are similar. We develop a general theoretical framework to describe this coupling, which allows us to retrieve the eigenfrequencies and mechanical loss of the bacterium vibration modes ( $Q$ factor). Finally, we analyse the effect of hydration on the vibrational properties of a single bacterium. This work demonstrates that ultrahigh frequency optomechanical resonators can be used for vibrational spectrometry with the unique capability to obtain information on single biological entities. 


\section{Introduction}

The measurement of the vibrational properties of molecules by optical inelastic scattering and optical absorption has allowed chemical identification of molecular species in a wide range of samples ${ }^{1}$. Raman spectroscopy is a paramount example of the power of this concept: a chemical make-up of the sample is obtained by measuring the frequency shift spectra of the inelastic scattering that corresponds to the frequencies of the vibration modes of the molecules within the sample. Frequency shifts range from hundreds of $\mathrm{GHz}$ to hundreds of $\mathrm{THz}$. Translation of this concept for identification and analysis of biomolecules has raised enormous interest. Before the first experimental realizations, theoretical studies had been predicted the existence of multiple low-frequency vibration modes in nucleic acid, proteins, virus and bacteria that are expected at the frontier between the $\mathrm{GHz}$ and $\mathrm{THz}$ regimes $^{2-6}$. These modes enclose valuable information on the flexibility that is sensitive to conformational changes, complexing with ligands and environmental cues ${ }^{7,8}$. It is now appreciated that many diseases are intimately connected with changes in the flexibility of biomolecules and biological systems ${ }^{9-11}$.

Figure $1 \mathrm{~A}$ shows the numerical calculation by the finite element method (FEM) of the fundamental resonance frequency of biological spherical particles adsorbed on a surface as a function of their radius. The eigenfrequencies of spherical particles go as $\sim \sqrt{\frac{E}{\rho}} \frac{1}{R}$, where $E$ is the Young's modulus, $\rho$ the density and $\mathrm{R}$ the radius ${ }^{4,6}$. We have used $E=1 \mathrm{GPa}$ and $\rho=1000 \mathrm{Kg} / \mathrm{m}^{3}$, that are representative values of biological particles in air conditions ${ }^{12,13}$. The data show that nanoscale biological particles such as proteins and viruses exhibit fundamental frequencies in the range of hundreds and tens of $\mathrm{GHz}$, respectively. Identification of these low-frequency vibration modes by optical inelastic scattering and optical absorption is extremely challenging ${ }^{14}$. Several experimental attempts have failed to find firm evidence of virus particle modes ${ }^{5,15}$. In the case of bacteria, mechanical resonances are expected at hundreds of megahertz, but their detection remains elusive 6,16. The simple existence of these underdamped mechanical resonances in biological particles such as viruses and bacteria remains an unsolved question, which ultimately needs to be addressed at the single particle level.

Here we propose the use of ultrahigh frequency mechanical resonators that can reach and surpass the frequency of the low-frequency vibration modes of bioparticles of size in the 
range of tens to hundreds of nanometers. In particular, we use optomechanical disk resonators supporting mechanical radial breathing modes (RBMs) and optical whispering gallery modes (WGM) (Fig 1B) $)^{17,18}$. A radial displacement of the disk modifies the electromagnetic conditions of the disk, shifting its WGM optical resonances and inducing a variation in the output optical intensity (Suppl. Mat.) RBMs exhibit very low mechanical energy dissipation when immersed in fluids, both in air and liquids, which enables to study biological entities near their native conformation ${ }^{19}$. The tiny Brownian fluctuations associated to the stiff RBMs can be resolved as consequence of the intense optomechanical coupling that provides a displacement noise floor of $\sim 10^{-18} \mathrm{~m} / \sqrt{ } \mathrm{Hz}^{20}$. Optomechanical disk resonators exhibit a combination of attributes such as ultrahigh resonance frequencies, large sensing area, ultrahigh displacement sensitivity and low mechanical energy dissipation, that place them as excellent devices to observe vibrational signatures of the coupling phenomena investigated here.

We note that micro- and nanomechanical resonators have been previously used for measuring the mass of single biological particles ${ }^{21,22,23}$. In addition to the mass, the stiffness of biological particles has been measured with compliant mechanical structures such as microcantilevers ${ }^{13,24}$. The added mass induces a downshift of the resonance frequency of the nanomechanical resonator, whereas the stiffness of the particle induces a upshift, usually smaller that the mass effect. The theory behind this response assumes that the biological particle is 'static', ignoring the thermal motion associated to the low-frequency vibration modes of the particle. Indeed, this has been a very correct approximation so far, because small particles feature vibration modes at much higher frequencies than those of the commonly used devices. However, what should we expect if we use mechanical resonators vibrating at frequencies near or above the mechanical resonances of the bioparticle? We address this question below.

We deposited single Staphylococcus epidermidis bacterium on optomechanical disks by means of an electrospray ionization system (Suppl. Mat.). Staphylococcus epidermidis are round-shaped bacteria with radius of $400 \pm 50 \mathrm{~nm}$. The fundamental radial breathing mode frequencies of the disks were measured in air before and after the bacteria deposition. Figure 1B shows two examples for $320 \mathrm{~nm}$ thick optomechanical disks of radius 5 and 2.5 $\mu \mathrm{m}$. The fundamental RBM frequencies of these disks, 272 and $546 \mathrm{MHz}$, respectively, are revealed in the amplitude frequency density of the Brownian fluctuations of the disk (Fig. 
1C). The quality factors were 3590 and 1640, respectively. The effect of the bacteria on the mechanical resonances of the disks is radically different. The resonance frequency of the larger disk is shifted to lower frequencies by $0.8 \%$, following the mass effect described above. A strikingly different behavior is observed in the smaller disk with higher frequency: the resonance frequency of the disk splits into two broad and close resonances with quality factors of 110 and 90 . This behavior is reminiscent of the frequency splitting observed when nearly identical mechanical resonators are coupled ${ }^{25-28}$. The resonance broadening also suggests a significant mechanical loss added by the presence of the bacterium.

\section{Theory of coupling between a mechanical resonator and an analyte}

We propose a lumped parameter system for describing the different scenarios that emerge in the interaction between a mechanical resonator and a bound analyte. Both structures support a quasi-infinite set of eigenmodes that form an eigenvector space. For the sake of simplicity, we restrict our analysis to the fundamental vibration mode of the sensor. Our system is thus described by a one-dimensional damped harmonic oscillator, associated to the sensor, that is coupled in series to $N$ damped harmonic oscillators vibrating along each of the directions of a $\mathrm{N}$-dimension function space formed by the eigenvectors of the analyte. Let $\hat{x}(\omega)=\left(\mathrm{x}_{\mathrm{S}}(\omega), \mathrm{x}_{\mathrm{A}, 1}(\omega), \mathrm{x}_{\mathrm{A}, 2}(\omega), \ldots, \mathrm{X}_{\mathrm{A}, \mathrm{N}}(\omega)\right)$ be the vector of the Fourier transform of the displacement of the sensor $\left(\mathrm{x}_{\mathrm{S}}\right)$ and analyte vibration modes $\left(\mathrm{x}_{\mathrm{A}, \mathrm{i}}\right)$. The displacement vector satisfies the motion equation given by,

$\left(-\omega^{2} \boldsymbol{M}+i \omega \boldsymbol{C}+\boldsymbol{K}\right) \widehat{\boldsymbol{x}}(\omega)=0$

where $\boldsymbol{M}, \boldsymbol{C}$ and $\boldsymbol{K}$ are the mass, damping and stiffness matrices that satisfy

$$
\begin{aligned}
& M_{i, j}=m_{S} \delta_{i 1} \delta_{1 j}+m_{A} \delta_{i j}\left(1-\delta_{1 j}\right) \\
& C_{i, j}=\left(\gamma_{S}+\sum_{k=1}^{\infty} \varphi_{k}^{2} \gamma_{A, k}\right) \delta_{i 1} \delta_{1 j}+\gamma_{A, i} \delta_{i j}\left(1-\delta_{1 j}\right)-\gamma_{A, j-1} \varphi_{j-1} \delta_{i 1}\left(1-\delta_{1 j}\right) \\
& K_{i, j}=\left(k_{S}+\sum_{l=1}^{\infty} k_{A, l} \varphi_{l}^{2}\right) \delta_{i 1} \delta_{1 j}+k_{A, i} \delta_{i j}\left(1-\delta_{1 j}\right)-k_{A, j-1} \varphi_{j-1} \delta_{i 1}\left(1-\delta_{1 j}\right)
\end{aligned}
$$

where $\delta_{i j}$ is the Kronecker delta; $k_{S}$ is the elastic constant associated to the sensor eigenfrequency, $k_{A, i}$ are the elastic constants associated to each of the analyte eigenfrequencies, $\omega_{S}$ is the sensor eigenfrequency, $\omega_{A, i}$ are the analyte eigenfrequencies, $m_{S}$ and $m_{A}$ are the effective mass of the sensor and analyte, and $\gamma_{S}$ and $\gamma_{A, i}$ are the damping coefficients of the sensor and analyte eigenmodes, respectively. The 
quality factor of the sensor and analyte vibration modes are given by the well-known relation $Q_{i, j}=\frac{m_{i} \omega_{i, j}}{\gamma_{i, j}}$. The coefficients $\varphi_{i}$ represent the projection of the fundamental eigenmode of the sensor onto the $i^{\text {th }}$ eigenvector of the analyte. Since the analyte eigenvectors form the basis of the function space, the coefficients $\varphi_{i}$ satisfy the condition $\sum_{i=1}^{N} \varphi_{i}{ }^{2}=1$. The eigenfrequency equation is given by $\operatorname{Det}\left(-\omega^{2} \boldsymbol{M}+i \omega \boldsymbol{C}+\boldsymbol{K}\right)=0$. The solution of the problem depends on the following dimensionless variables: the ratio of the analyte mass to the sensor mass, $\mu=\frac{m_{A}}{m_{S}}$, the relative frequency defined as $\Omega=\frac{\omega}{\omega_{S}}$, the ratio of the sensor eigenfrequency to the fundamental eigenfrequency of the analyte, $\Lambda=\frac{\omega_{S}}{\omega_{A, 1}}$, and the ratio between the higher eigenfrequencies of the analyte and its fundamental resonance frequency, $\beta_{n}=\frac{\omega_{A, n}}{\omega_{A, 1}}$.

The complexity of the solutions of the coupled mechanical system exponentially increases with $N$. We analyze here the case $N=2$, i.e., two orthogonal analyte springs in series with the sensor spring (Fig. 2A, inset). As shown below, this model allows describing the different scenarios that can be found when an analyte lands on the surface of a nanomechanical resonator. The solution for the complex eigenfrequencies is described in the Suppl. Mat. Figure 2 shows the stochastic response of the sensor for a case where $\mu=$ $0.05, \beta_{2}=4.2, Q_{S}=1000, \varphi_{1}=0.8, Q_{A, 1}=20, Q_{A, 2}=10$. These values can be expected when a viscoelastic bioparticle lands on mechanical resonators with high Q-factor. Independently of the precise values used for the parameters of the problem, the stochastic frequency response of the sensor upon analyte attachment exhibits three regimes: the inertial regime when $\Lambda \ll 1$, the coupling regime when $1 \lesssim \Lambda \lesssim \beta_{2}$ and the insensitive regime when $\Lambda \gg \beta_{2}$. We begin by analyzing the asymptotic regimes, $\Lambda \ll 1$ and $\Lambda \gg \beta_{2}$. In the inertial regime, the sensor cannot follow the thermal fluctuations of the analyte that are located at frequencies much higher than the resonance frequency of the sensor. The sensor resonance frequency is given by the well-known relation $\Omega=\frac{1}{\sqrt{1+\mu}}$ that describes the resonance frequency decrease due to the mass of a 'static' analyte. Notice that in our model, the sensor mode and the analyte vibration modes are in series, and therefore we neglect the stiffness effect. This assumption is a good approximation in most of the cases and leads to tractable analytical solutions. At the other extreme of the frequency spectrum, we find the insensitive regime, in which the sensor resonance frequency is significantly higher than the highest vibration mode of the analyte that couples with the sensor. Since 
the sensor vibration cannot be followed by the floppy analyte, the sensor resonance frequency remains unperturbed by the analyte.

The coupling regime settles between the asymptotic inertial and insensitive regimes. In this regime, the stochastic vibrations of the sensor and the analyte exhibit a significant spatiotemporal coherence. When the resonance frequency of the sensor is close to an analyte eigenfrequency, i.e. $\Lambda \approx 1$ or $\Lambda \approx \beta_{2}$, the vibration modes of the sensor and analyte become hybridized, giving rise to the emergence of coupled modes. The minimum difference between the eigenfrequencies of the coupled modes is $\varphi_{i} \sqrt{\mu}$, i.e., $\varphi_{1} \sqrt{\mu}$ and $\sqrt{1-\varphi_{1}^{2}} \sqrt{\mu}$, respectively. The parameter $\varphi_{i} \sqrt{\mu}$ referred to as the coupling constant in classical problems of coupled harmonic oscillators, describes the strength of the interaction between the sensor fundamental mode and the corresponding vibration mode of the analyte. In these conditions of optimal coupling, the $Q$-factor of the coupled modes is

approximately given by $\frac{2 Q_{S} Q_{A, i}}{Q_{S}+Q_{A, i}}$. The major contribution to the mechanical loss arises from the analyte, which significantly dissipates more energy than the sensor. The sensor resonance frequency is little modified between the regions of highest coupling, varying from positive to negative variations. In this region, the sensor frequency response cannot be related to the added mass. Importantly, the expressions given here and in the Suppl. Mat. for $\mathrm{N}=2$ can be generalized for more modes as long, as the analyte modes are uncoupled with each other. This condition is largely satisfied in the limit of small dissipation, i.e., $Q_{A, i}>>1$.

\section{Validation of the theory with numerical simulations}

The validity of the analytical model shown in the previous section was checked by performing numerical calculations by the finite element method (FEM). In particular, we have calculated the mechanical response of the disk with the attached bacterium shown in Fig. 1B (case $\left.{ }^{\star *}\right)$. The geometry and dimensions of the disk structure and attached bacterium were accurately reproduced and finely meshed in the FEM simulations. The properties of the material disk are known. However, the bacterium properties are unknown. Frequency measurements in the inertial regime were performed with microcantilever resonators to obtain the mass of the bacterium ${ }^{13}$. Correlation of these measurements with SEM images provides a density of about $920 \mathrm{~kg} / \mathrm{m}^{3}$. As a first approximation, we fix the Poisson ratio to 0.35 , leaving the Young's modulus of the bacterium as the free parameter. 
Figure $3 \mathrm{~A}$ shows the first four eigenfrequencies of the bacterium attached to an infinitely rigid substrate. The first and third vibration modes arise from the attachment of the bacterium to the solid support, thereby they do not exist when the bacterium is free in suspension. In the first mode, the bacterium is subject to bending deformation, whereas in the third mode the bacterium elongates and contracts periodically in the vertical direction. Second and fourth modes are modifications of the vibration modes of quasispherical particles in suspension observed by inelastic light scattering ${ }^{29}$. In particular, they respectively correspond with the fundamental radial breathing mode and the quadrupolar mode, where the sphere deforms into transient oblate and prolate shapes over consecutive half periods of vibration. By varying the bacterium Young's modulus, we can sweep the ratio of the sensor eigenfrequency to the fundamental eigenfrequency of the analyte $(\Lambda)$ almost two decades. The numerical calculations of the disk eigenfrequency as a function of $\Lambda$ are shown in Fig. 3B. The most significant coupling region takes place when the disk eigenfrequency is close to the first bacterium vibration mode, $\Lambda \approx 1$. In this situation, the radial motion of the disk is efficiently translated into the bacterium bending through the stress generated at the contact area. The experimental data (Fig. 1C, case ${ }^{\star *}$ ) match this coupling region for a Young's modulus of the bacterium of about $5.5 \mathrm{GPa}$, which is consistent with the values found in the literature for dry bacteria and viruses ${ }^{13,30}$. In this coupling region, the RBM of the disk splits into two collective eigenmodes, in which the bacterium bends in phase and antiphase with the radial motion of the disk. Mechanical coupling of the disk with either the second or third vibration mode of the bacterium is not observed $\left(\Lambda \approx \beta_{2}\right.$ or $\left.\Lambda \approx \beta_{3}\right)$, which implies that $\varphi_{2}$ and $\varphi_{3}$ are negligible. These results can be explained by examining the vibration mode shapes. For instance, the second vibration mode of the bacterium can be driven by radial vibration at the contact surface, however this can be only achieved if the bacterium is sitting just on the center of the disk, which is not the case in our experiment. Moreover, the third vibration mode cannot be coupled as it involves deformation of the bacterium in the orthogonal direction to the radial deformation of the disk. On the other hand, the numerical calculations show a small coupling behavior when the disk eigenfrequency is close to the fourth bacterium vibration mode. The FEM results are fitted to the analytical model described above. Our model assumes that the analyte only features two vibration modes that satisfy the orthogonal condition, in this case, $\varphi_{1}{ }^{2}+\varphi_{4}{ }^{2}=1$. In order to be more realistic, we relax this constraint by imposing instead $\varphi_{1}{ }^{2}+\varphi_{4}{ }^{2}<1$, thus assuming the existence of more vibration modes, but weakly coupled 
to the sensor. Solid line in Fig. 3B represents the fitting of the model to the numerical simulations. We observe a significantly good agreement between the numerical and analytical data. The fitting parameters are $\varphi_{1}=0.75$ and $\varphi_{4}=0.4$. The relative simplicity of our model allows the development of efficient algorithms for retrieving the eigenfrequency and Q-factor of the bacterium (Suppl. Mat.). The application of these algorithms to the experimental data (Fig. 1C, case $^{\star *}$ ) provides a resonance frequency of the bacterium bending mode of $0.541 \pm 0.001 \mathrm{GHz}$ and a $\mathrm{Q}$-factor of $44 \pm 4$. These data represent the first measurement of a low-frequency vibration mode of a single biological particle.

\section{Monitoring bacterium hydration by measuring its eigenfrequency and quality factor}

Figure 4A shows an event where a bacterium has adsorbed at the disk's edge. We measured the frequency response of the system in an environmental chamber where the relative humidity could be varied. It is known that mass and viscoelasticity of biopolymers critically depend on the hydration level ${ }^{31,32}$. However, very little is known about how humidity affects to microbial particles. It is estimated that bacterium mass can increase about $20 \%$ when the relative humidity increases from near zero to about $80 \% 33,34$. The water uptake by the bacterium leads to significant changes in its mechanical properties ${ }^{35-37}$. The stiffness of bacteria is largely determined by the viscoelastic properties of the cell wall, consisting of a network of peptidoglycan polymers. As many other biopolymers, the cell wall behaves like a glassy polymer in dry conditions that softens with hydration to behave like a rubbery polymer at wet conditions ${ }^{38}$. The frequency spectrum of the disk as a function of the relative humidity is plotted in Fig. 4B. As shown above, the fundamental resonance peak of the disk splits into two broad resonance peaks. Second peak is significantly broader than the first one. Assuming that mechanical losses are higher in the bacterium than in the disk, we can conclude that these two collective vibration modes exhibit mechanical localization in the disk in the first mode and in the bacterium in the second mode. FEM simulations show that in this geometrical configuration, the most significant coupling occurs when the disk eigenfrequency is close to the extensional third vibration mode of the bacterium shown above (Suppl. Mat.). The disk RBM efficiently induces extensional motion of the bacterium in the radial direction. The frequency and Q-factor of the coupled modes are plotted in Fig. 4C. At humidity less than $25 \%$, the eigenfrequencies and $Q$-factors remain approximately constant, and then fall with a further increase in humidity. The eigenfrequency decrease is 
small, reaching $0.7 \%$ and $1.4 \%$ for the first and second vibration modes. In contrast, the mechanical dissipation increases significantly with humidity. The Q-factors of the first and second coupled modes drops $45 \%$ and $15 \%$, respectively. In addition, the difference between the Q-factors decreases, which implies that the delocalization of the coupled modes increases.

The data are made more understandable by solving the inverse problem for obtaining the frequency and quality factor of the bacterium vibration mode (Fig. 4D). The bacterium eigenfrequency follows the same trend than the coupled eigenfrequencies. It is insensitive to humidity up to $25 \%$, then falls up to a decrease of $2 \%$ near $80 \%$ relative humidity. The most significant change again is related to the energy dissipation. The $Q$-factor of the bacterium decreases by $30 \%$ from the dry to the wet state. The significant $Q$-factor reduction is expected from the viscoelastic properties of the bacterium (Suppl. Mat.). However, the bacterium eigenfrequency changes are significantly smaller, suggesting that the eigenfrequency of the bacterium is little sensitive to the environmental conditions. A tentative explanation is that the vibration mode of the bacterium mainly depends on the dry structure. Characteristic relaxation times of liquids, such as water, range from $\sim 1$ ps for free water to $\sim 1$ ns for bound water, as it is the case here. Our data suggest that the water adsorbed on the bacterium is unable to relax at the ultrahigh frequencies of the disk. This hypothesis is in consistency with recent literature ${ }^{19,39,40}$. We remark that our knowledge on the mechanical properties of biomaterials at ultrahigh frequencies is still scarce, and more studies are needed to understand environmental effects on single cell and single pathogen entities. The presented methodology allows studying the interaction of the bacterium with the environment.

\section{Conclusions}

We have demonstrated that ultrahigh frequency optomechanical resonators can be used to measure the low-frequency vibration modes of biological systems, such as bacteria, at the single entity level. With these results we take mechanical sensing beyond the wellestablished mass and stiffness measurements. We foresee that these results will enable a novel class of biological spectrometry for fingerprinting biological particles by their vibrational properties. The technology presented here is still limited, as the device eigenfrequencies must be close to the vibration frequencies of the analyte. A general applicability of this technology will require the development of highly tunable 
optomechanical resonators that can be coupled to the bioanalyte of interest. If this is achieved, optomechanical spectrometry could lead to advances in microbiomics, as well as for label-free diagnosis of infectious diseases.

\section{REFERENCES}

1 Smith, E. \& Dent, G. Modern Raman Spectroscopy: A Practical Approach. (Wiley, 2019).

2 Davydov, A. S. The theory of contraction of proteins under their excitation. J. Theor. Biol. 38, 559-569 (1973).

3 Hay, S. \& Scrutton, N. S. Good vibrations in enzyme-catalysed reactions. Nat. Chem. 4, 161168 (2012).

4 Ford, L. H. Estimate of the vibrational frequencies of spherical virus particles. Phys. Rev. E 67, 051924 (2003).

5 Sirotkin, S., Mermet, A., Bergoin, M., Ward, V. \& Van Etten, J. L. Viruses as nanoparticles: structure versus collective dynamics. Phys. Rev. E 90, 022718 (2014).

6 Zinin, P., Allen III, J. S. \& Levin, V. Mechanical resonances of bacteria cells. Phys. Rev. E 72, 061907 (2005).

7 Turton, D. A. et al. Terahertz underdamped vibrational motion governs protein-ligand binding in solution. Nat. Commun. 5, 3999 (2014).

8 Acbas, G., Niessen, K. A., Snell, E. H. \& Markelz, A. G. Optical measurements of long-range protein vibrations. Nat. Commun. 5, 3076 (2014).

9 Longo, G. et al. Antibiotic-induced modifications of the stiffness of bacterial membranes. $J$. Microbiol. Meth. 93, 80-84 (2013).

10 Köhler, J. et al. Mutation of the myosin converter domain alters cross-bridge elasticity. Proc. Natl. Acad. Sci. USA 99, 3557-3562 (2002).

11 Greber, U. F. Virus and host mechanics support membrane penetration and cell entry. J. Virol. 90, 3802-3805 (2016).

12 Roos, W. H., Bruinsma, R. \& Wuite, G. J. L. Physical virology. Nat. Phys. 6, 733-743 (2010).

13 Malvar, O. et al. Mass and stiffness spectrometry of nanoparticles and whole intact bacteria by multimode nanomechanical resonators. Nat. Commun. 7, 13452 (2016).

14 Dexheimer, S. L. Terahertz Spectroscopy: Principles and Applications. (CRC Press, 2007).

15 Stephanidis, B., Adichtchev, S., Gouet, P., McPherson, A. \& Mermet, A. Elastic properties of viruses. Biophys. J. 93, 1354-1359 (2007).

16 Zinin, P. V. \& Allen III, J. S. Deformation of biological cells in the acoustic field of an oscillating bubble. Phys. Rev. E 79, 021910 (2009).

17 Favero, I. \& Karrai, K. Optomechanics of deformable optical cavities. Nat. Photonics 3, 201205 (2009).

18 Ding, L. et al. High frequency GaAs nano-optomechanical disk resonator. Phys. Rev. Lett. 105, 263903 (2010).

19 Gil-Santos, E. et al. High-frequency nano-optomechanical disk resonators in liquids. Nat. Nanotechnol. 10, 810-816 (2015).

20 Baker, C. et al. Photoelastic coupling in gallium arsenide optomechanical disk resonators. Opt. Express 22, 14072-14086 (2014).

21 Hanay, M. S. et al. Single-protein nanomechanical mass spectrometry in real time. Nat. Nanotechnol. 7, 602-608 (2012).

22 Dominguez-Medina, S. et al. Neutral mass spectrometry of virus capsids above 100 megadaltons with nanomechanical resonators. Science 362, 918-922 (2018).

23 Liu, F., Alaie, S., Leseman, Z. C. \& Hossein-Zadeh, M. Sub-pg mass sensing and measurement with an optomechanical oscillator. Opt. Express 21, 19555-19567 (2013). 
24 Kosaka, P. M., Calleja, M. \& Tamayo, J. Optomechanical devices for deep plasma cancer proteomics. Semin. Cancer Biol. 52, 26-38 (2018).

25 Spletzer, M., Raman, A., Wu, A. Q., Xu, X. \& Reifenberger, R. Ultrasensitive mass sensing using mode localization in coupled microcantilevers. Appl. Phys. Lett. 88, 254102 (2006).

26 Gil-Santos, E. et al. Mass sensing based on deterministic and stochastic responses of elastically coupled nanocantilevers. Nano Lett. 9, 4122-4127 (2009).

27 Gil-Santos, E., Ramos, D., Pini, V., Calleja, M. \& Tamayo, J. Exponential tuning of the coupling constant of coupled microcantilevers by modifying their separation. Appl. Phys. Lett. 98, 123108 (2011).

28 Stassi, S. et al. Large-scale parallelization of nanomechanical mass spectrometry with weakly-coupled resonators. Nat. Commun. 10, 3647 (2019).

29 Duval, E. Far-infrared and Raman vibrational transitions of a solid sphere: Selection rules. Phys. Rev. B 46, 5795-5797 (1992).

30 Ruz, J. J., Tamayo, J., Pini, V., Kosaka, P. M. \& Calleja, M. Physics of nanomechanical spectrometry of viruses. Sci. Rep. 4, 6051 (2014).

31 Furusawa, H., Sekine, T. \& Ozeki, T. Hydration and viscoelastic properties of high-and lowdensity polymer brushes using a quartz-crystal microbalance based on admittance analysis (QCM-A). Macromolecules 49, 3463-3470 (2016).

32 Domínguez, C. M. et al. Effect of water-DNA interactions on elastic properties of DNA selfassembled monolayers. Sci. Rep. 7, 536 (2017).

33 Bateman, J. B., Stevens, C. L., Mercer, W. B. \& Carstensen, E. L. Relative humidity and the killing of bacteria: the variation of cellular water content with external relative humidity or osmolality. Microbiology 29, 207-219 (1962).

34 Rubel, G. O. Measurement of water vapor sorption by single biological aerosols. Aerosol Sci. Tech. 27, 481-490 (1997).

35 Deng, Y., Sun, M. \& Shaevitz, J. W. Direct measurement of cell wall stress stiffening and turgor pressure in live bacterial cells. Phys. Rev. Lett. 107, 158101 (2011).

36 Nikiyan, H., Vasilchenko, A. \& Deryabin, D. Humidity-dependent bacterial cells functional morphometry investigations using atomic force microscope. Int. J. Microbiol. 2010, 704170 (2010).

37 Thwaites, J. J. \& Surana, U. C. Mechanical properties of Bacillus subtilis cell walls: effects of removing residual culture medium. J. Bacteriol. 173, 197-203 (1991).

38 Kulasinski, K., Guyer, R., Keten, S., Derome, D. \& Carmeliet, J. Impact of moisture adsorption on structure and physical properties of amorphous biopolymers. Macromolecules 48, 27932800 (2015).

39 Guillet, Y., Abbas, A., Ravaine, S. \& Audoin, B. Ultrafast microscopy of the vibrational landscape of a single nanoparticle. Appl. Phys. Lett. 114, 091904 (2019).

40 Hsueh, C.-C., Gordon, R. \& Rottler, J. Dewetting during terahertz vibrations of nanoparticles. Nano Lett. 18, 773-777 (2018).

\section{ACKNOWLEDGEMENTS}

This work was supported by the European Union's Horizon 2020 research and innovation program under grant agreement No 731868 - VIRUSCAN and European Research Council Grant 681275 - LIQUIDMASS- ERC- CoG-2015 and 770933-NOMLI-ERC-CoG 2017, and by the Spanish Science, Innovation and Universities Ministry through project CELLTANGLE reference RTI2018-099369-B-I00 and Ramón y Cajal grant RYC-2017-21640 to P. M. K.; by the Comunidad de Madrid (iLUNG B2017/BMD-3884) with support from EU (FEDER, FSE). We acknowledge Prof. J. Mingorance for guidance and for providing the bacterial samples. All authors acknowledge service from IMN X-SEM Laboratory, which is funded by 
MCIU (project CSIC13-4E-1794) and EU (FEDER, FSE). E.G.S. acknowledges financial support by Fundación General CSIC (Programa ComFuturo), as well as Marie-Sklodowska Curie Actions (H2020-MSCA-IF-2015) under NOMBIS project (703354).

\section{AUTHOR CONTRIBUTIONS}

E.G., M.C. and J.T. conceived and designed the experiments: E. G. and O. M. performed the experiments: E.G., J. R. and J. T. analyzed the data and develop the theory: S. G., O. M. and P. K. contributed materials and tools to deposit bacteria: E. G., A. L. and I. F. designed and fabricated the devices: J. T., M. C. and E. G. co-wrote the paper. All authors discussed the results and commented on the manuscript.

\section{DATA AVAILABILITY STATEMENT}

The data that support the plots within this paper and other findings of this study are available from the corresponding author upon reasonable request

\section{ADDITIONAL INFORMATION}

Supplementary information is available in the online version of the paper. Reprints and permission information is available online at www.nature.com/reprints. Correspondence and requests for materials should be addressed to E. G. and J. T. 

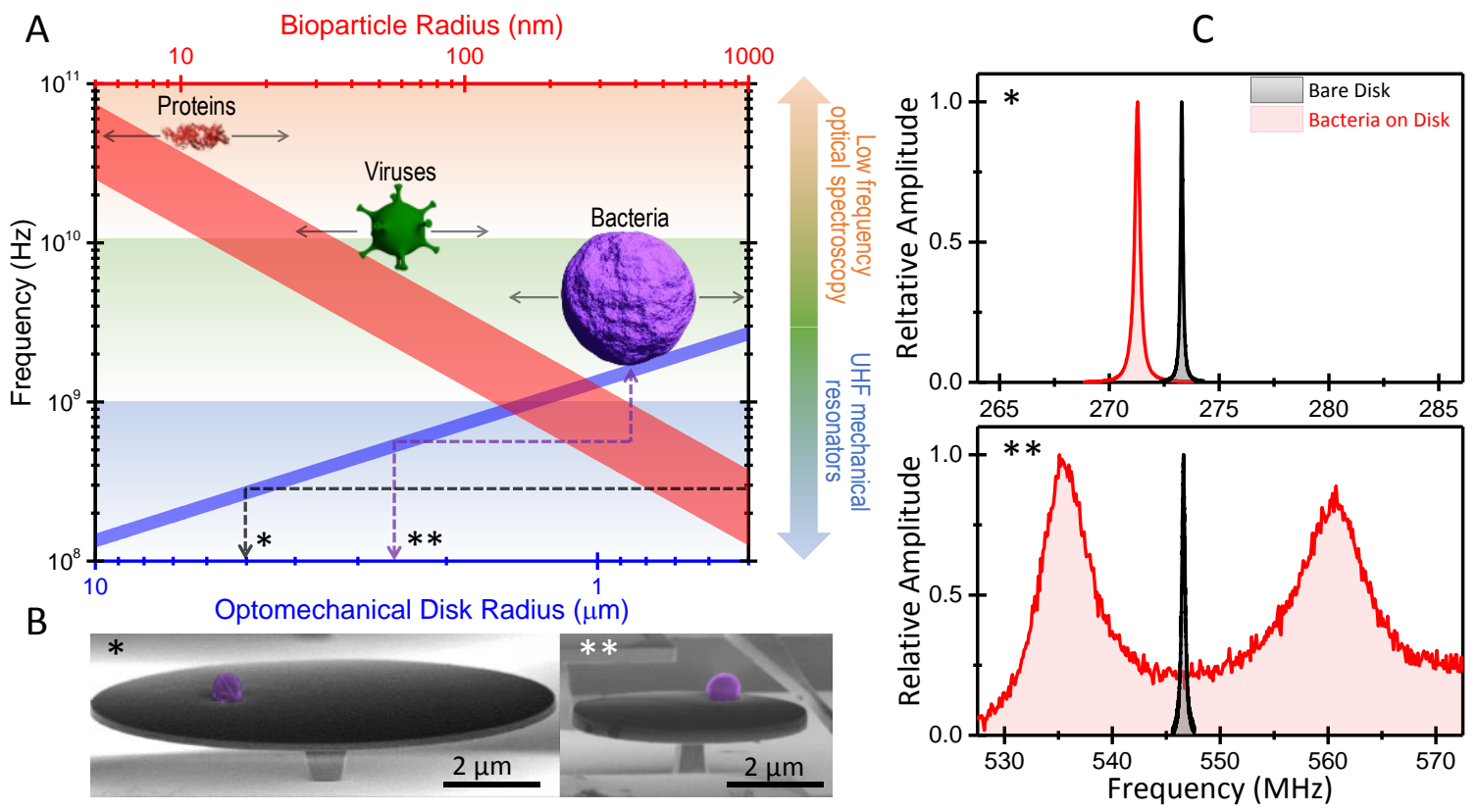

Fig. 1 Fundamental vibration modes of biological particles and optomechanical disk resonators at the gigahertz regime. A. Frequency of the radial breathing mode of a $320 \mathrm{~nm}$ thick optomechanical disk (blue region) and of the fundamental mode of quasi-spherical biological particles adsorbed on a rigid support (red region), as a function of the disk and bioparticle radii, respectively.. Staphylococcus epidermidis are round-shaped bacteria with radius of $400 \pm 50 \mathrm{~nm}$ and expected fundamental frequency of $500 \pm 100 \mathrm{MHz}$. Optomechanical disk resonators with radius of 2-3 $\mu \mathrm{m}$, marked as ${ }^{* *}$, show resonance frequencies converge to the same range. B. Scanning electron microscopy of two optomechanical disk resonators with radii $5 \mu \mathrm{m}$ (image marked with *) and $2.5 \mu \mathrm{m}$ (image marked with ${ }^{* *}$ ) with an attached S. epidermidis cell. C. Effect of bacterium adsorption on the radial breathing mode of the optomechanical disks marked with * and ${ }^{* *}$ shown in B.. 

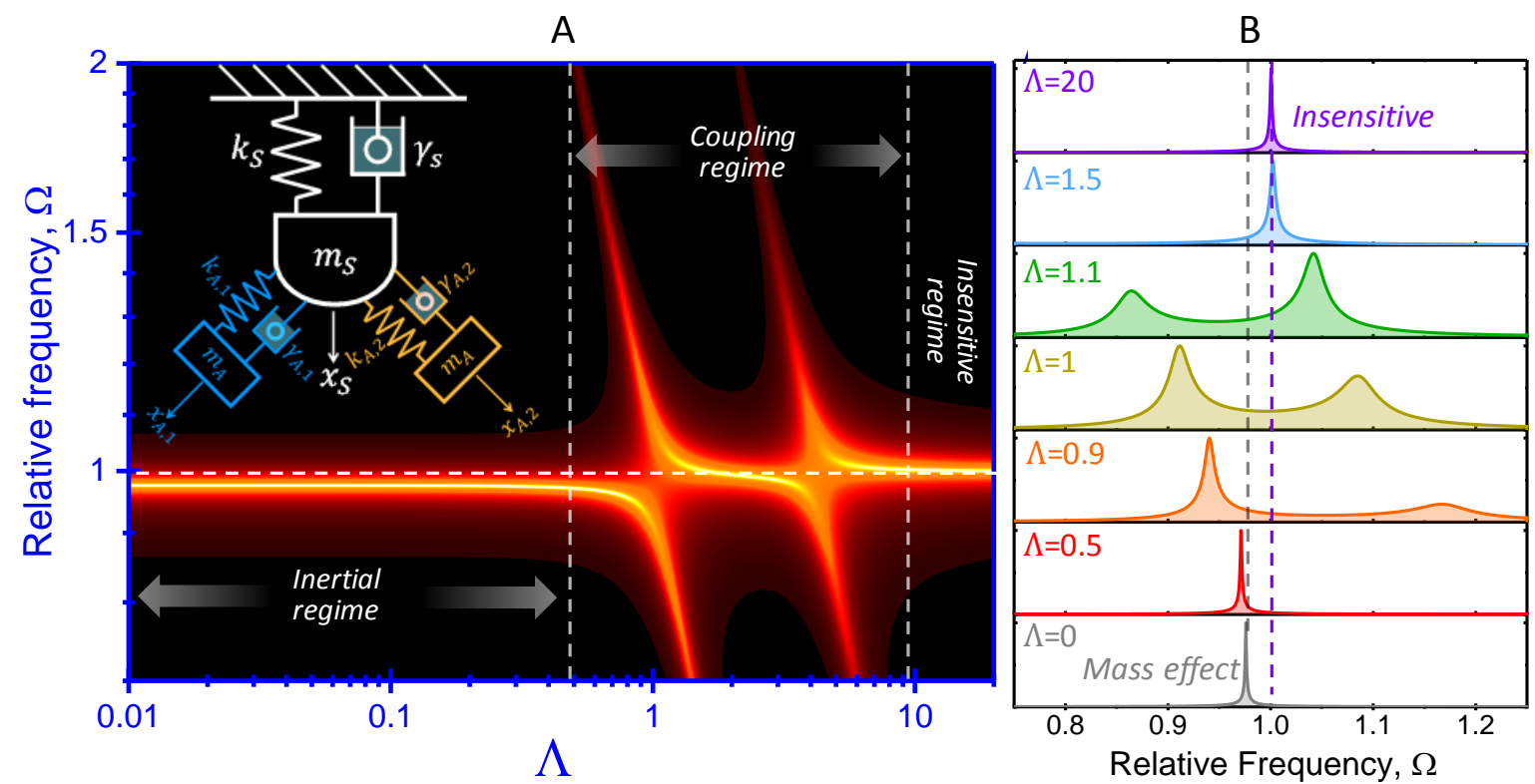

Fig. 2 Stochastic response of a sensing damped harmonic oscillator coupled in series to an analyte that exhibits two orthogonal vibration modes, represented as two damped harmonic oscillators. A. Color-intensity map of the amplitude of the thermal fluctuations of the sensor as a function of the dimensionless frequency and the ratio of the sensor eigenfrequency to the fundamental eigenfrequency of the analyte $(\Lambda)$. The graph shows the three regimes that can be found in the frequency response of a mechanical resonator upon attachment of an analyte: the inertial regime, the coupling regime and the insensitive regime. The inset shows the corresponding lumped parameter model used in the calculations. B. Cross-sections of the graph A for several values of $\Lambda$. Grey and purple vertical dashed lines represent the fundamental resonance frequencies of the unloaded optomechanical disk and loaded disk in the inertial regime, respectively. 
A

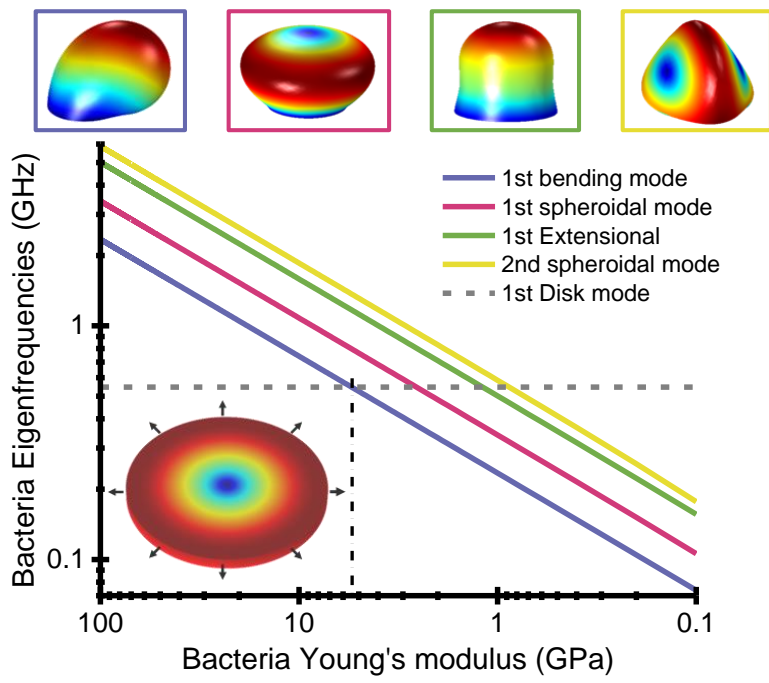

B

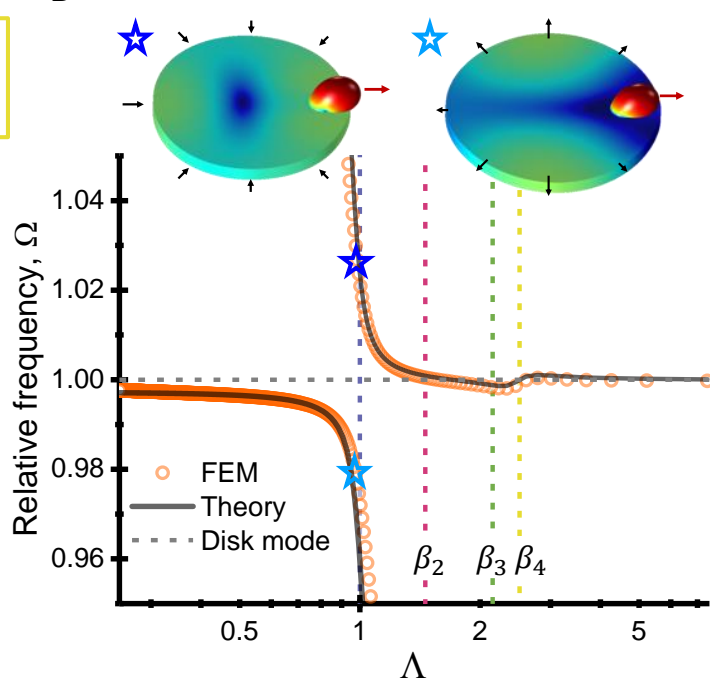

Fig. 3 Numerical calculation of the mechanical coupling of the bacterium and the disk resonator. A FEM calculation of the four eigenfrequencies of a $800-\mathrm{nm}$ diameter quasispherical particle attached to an infinitely rigid support as a function of the Young's modulus. Poisson's ratio and density are fixed to 0.35 and $920 \mathrm{~kg} / \mathrm{m}^{3}$, respectively. The contact radius is $300 \mathrm{~nm}$. The threedimensional vibration mode shapes of the bacterium are shown at the top in colour-coded boxes. Vertical dot-dash line represents the Young's modulus of the bacteria where the $1^{\text {st }}$ bending mode matches the frequency of the fundamental radial breathing mode of the disk (horizontal grey dash line). B. FEM calculation of the eigenfrequency of a disk with the quasispherical particle attached near its edge (solid circles) as a function of the ratio of the sensor eigenfrequency to the fundamental eigenfrequency of the analyte, $\Lambda$, is obtained by varying the Young's modulus of the bacterium (A). The star symbols represent the experimental data shown in Fig. 1C, case ${ }^{* *}$. The vibration mode shapes in this coupling state are shown at the top. Colors represent the normalized displacement in logarithmic scale. Vertical color dashed lines represent the ratio between the higher eigenfrequencies of the bacterium and its fundamental resonance frequency $\left(\beta_{n}\right)$. Horizontal dashed grey line represents the disk eigenfrequency. Solid line represents the fitting of FEM data to the analytical model. 

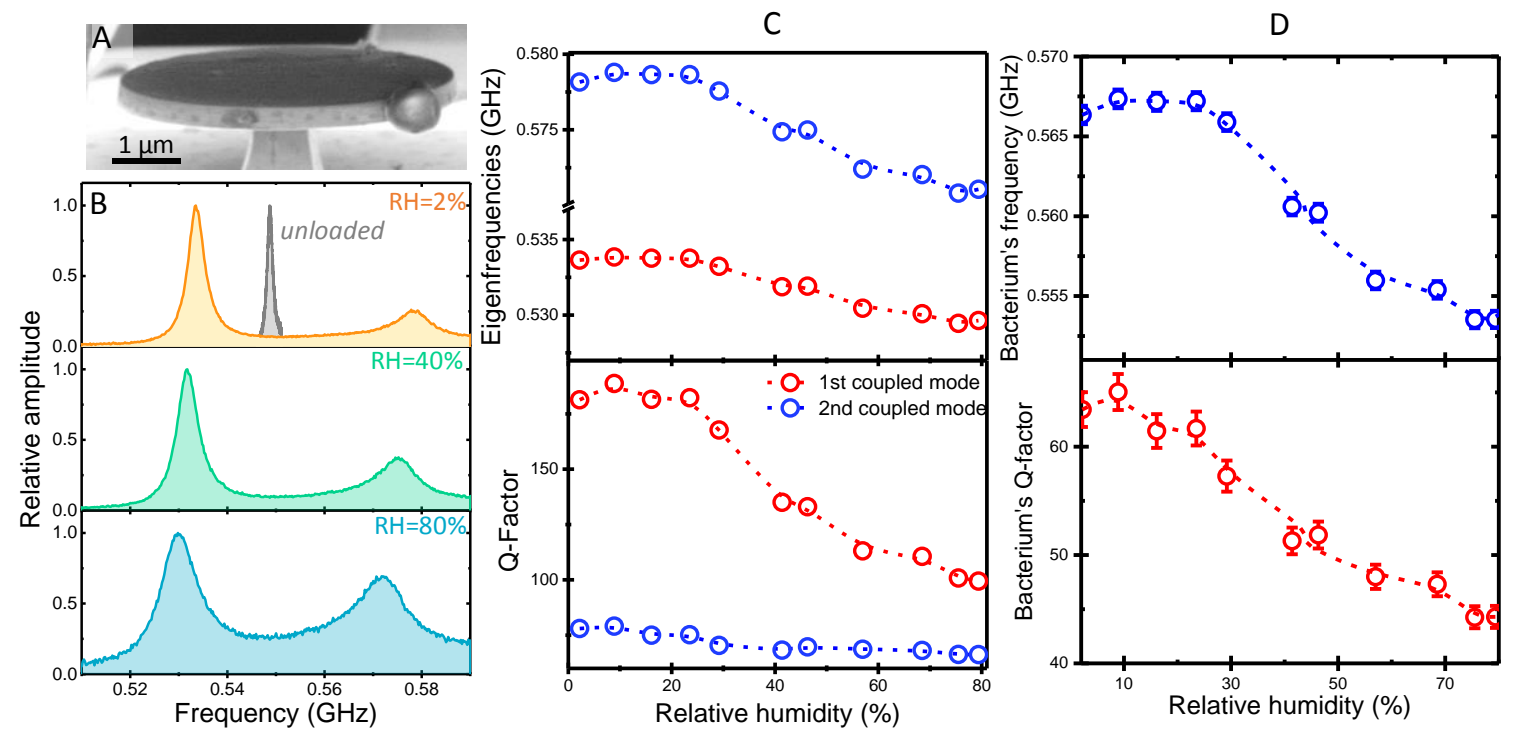

Fig. 4 Effect of hydration on the vibration mode of a bacterium. A. Scanning electron microscopy image of a GaAs UHF disk of radius $2.5 \mu \mathrm{m}$ with an adsorbed bacterium on the edge. B. Brownian frequency spectra of the disk-bacterium system for three representative values of the relative humidity $(\mathrm{RH})$. The acquisition time of each spectra is $1 \mathrm{~s}$ and each spectra is averaged 100 times. The resonance frequency and Q-factor of the unloaded disk are $0.59 \mathrm{GHz}$ and 440, respectively C. Eigenfrequencies and Q-factors of the coupled modes as a function of the relative humidity (symbols) obtained by fitting the frequency spectra to the superposition of two independent damped harmonic oscillators. Dashed line represents the spline interpolation. D. Bacterium's eigenfrequency and Qfactor obtained by applying the inverse problem algorithm. The error bars arise from the errors obtained in the fitting of the frequency spectra (not shown in $C$ as they are smaller than the symbol size). These errors, particularly the error in the Q-factors, propagate in the inverse-problem calculation giving rise to noticeable errors in eigenfrequency and Q-factor of the bacterium. 